Bull. Mater. Sci., Vol. 36, No. 1, February 2013, pp. 15-24. (c) Indian Academy of Sciences.

\title{
Investigation of magnetically enhanced swelling behaviour of superparamagnetic starch nanoparticles
}

\author{
A K BAJPAI* and SWETA LIKHITKAR \\ Department of Chemistry, Government Autonomous Science College, Jabalpur 482 002, India
}

MS received 13 June 2011; revised 20 September 2011

\begin{abstract}
The present study follows a novel strategy for the preparation of superparamagnetic nanoparticles of cross-linked starch impregnated homogeneously with nanosized iron oxide. The prepared magnetic nanoparticles were characterized by infra-red (FTIR) spectroscopy, transmission electron microscopy (TEM) and X-ray diffraction and magnetization studies. The size of the magnetic polymeric particles was found to lie in the range of 20-80 $\mathrm{nm}$, and they exhibited superparamagnetic properties. The particles were allowed to swell in phosphate buffer saline (PBS) and the influence of factors such as chemical composition of nanoparticles, $\mathrm{pH}$ and temperature of the swelling bath and applied magnetic field was investigated on the water intake capacity of the nanoparticles. The prepared nanoparticles showed potential to provide a possible option for controlled and targeted delivery of anticancer drugs, applying external magnetic field.
\end{abstract}

Keywords. Starch; iron oxide; nanotechnology; nanoparticles; superparamagnetic; drug delivery.

\section{Introduction}

The in vitro applications of nanoparticles in diagnostics have been practised for nearly 40 years. This is due to a number of beneficial factors of nanoparticles that include a large surface area to volume ratio and the possibility of ubiquitous tissue accessibility (Saboktakin et al 2009).

The most widely known and extensively used magnetic nanoparticles are ferro fluids due to the advantages they have in comparison to other particles: they are compatible with living organisms, present an excellent in vivo stability, and migrate easily under magnetic fields. These nanoparticles were clinically tested with promising results. Magnetic nanosized particles have already been known for over 50 years, but research into their potential use in medicine and pharmaceutics is of recent origin (Liu et al 2004; Arruebo et al 2007). Since 1996, a great deal of research has been carried out in designing drug delivery systems where drugs were found chemically bounded in these nanoparticles having diameters around $100 \mathrm{~nm}$ (Lüebbe et al 2001), and these nanomaterials also exhibit very interesting electrical, optical, magnetic and chemical properties which cannot be achieved by their bulk counterparts (Nidhin et al 2008).

Major applications of these magnetic materials include magnetic field-guided drug delivery, magnetic cell separations and magnetic hyperthermia therapy for treatment of tumors (Honda et al 1998; Gomez-Lopera et al 2001; Kusaka et al 2002; Roullin et al 2002; Kim et al 2003; Liu et al 2004). The unique combination of high magnetization and

*Author for correspondence (akbmrl@yahoo.co.in, akbajpailab@yahoo.co.in) paramagnetic behaviour opens these materials to a very wide range of biomedical and pharmaceutical applications. Particularly, the possibilities of nanoparticles modification by biologically active compounds to use them in controlled drug delivery systems, as agents in magnetic resonance imaging and for magnetic induced tumor treatment via hyperthermia are very interesting (Kumar 2005).

Starch is a natural, renewable and biodegradable polymer produced by many plants as a source of stored energy (Corre et al 2010). It is an abundant biodegradable and inexpensive polysaccharide and unique amongst carbohydrates because it occurs naturally as discrete particles, called granules. The granules are the primary means of energy storage in green plants over long periods of time, and the shape and size of the granules depend on their origin (Fang and Fowler 2002; Lehr et al 2010). Starch is being used as a biodegradable polymer. Moreover, the use of starch microspheres has also been suggested for immobilization, parenteral and nasal administration (Parikh et al 2003; Conte et al 2005). A number of multifunctional crosslinking reagents have been suggested for starch and in them epichlorohydrin finds wide use.

The rational of using nanoparticles for tumor targeting is based on the fact that the nanoparticles will be able to deliver a concentrate dose of drug in the vicinity of the tumor targets via the enhanced permeability and retention effect or active targeting by ligands on the surface of nanoparticles (Mohanraj and Chen 2006; Balaban et al 2003). One of the prime factors regulating the extent of drug loading depends on hydrophilic nature of the carrier that offers ability to imbibe large amounts of drug solution when left in the drug reservoir. Thus, water sorption capacity of nanocarriers 
is a significant property that deserves careful investigation. Thus, being motivated by the importance of water sorption property of nanocarriers, the present study aims at designing iron oxide impregnated starch nanoparticles possessing superparamagnetic behaviour. Although there are many approaches available to prepare iron oxide containing polymer nanocomposites, however, the major problem that is frequently encountered is lack of homogeneous dispersion of inorganic moieties within the polymer matrix. This is a significant point which deserves attention also as the superparamagnetic behaviour and allied magnetic properties and performance of the nanocomposite greatly depends on how uniformly the magnetic particles have been impregnated inside the bulk of the host polymer.

In the present study an original and novel approach has been adopted to impregnate iron oxide nanoparticles into the matrix of starch nanoparticles. The approach involves soaking of $\mathrm{Fe}^{2+} / \mathrm{Fe}^{3+}$ ions into the starch nanoparticles matrix and subsequent precipitation of iron as iron oxide. This method has advantage in the sense that using this approach uniformly impregnated iron oxide nanoparticles may be achieved within the starch matrix.

\section{Experimental}

\subsection{Materials}

Water-soluble starch was purchased from E. Merck, Mumbai, India and used without any pretreatment. Epichlorohydrin was employed as a crosslinker for starch and supplied by Thomas Baker Chemicals, Mumbai, India and used as received. Silicon oil was used as an oil phase and purchased from Research Lab, Mumbai, India. $\mathrm{FeCl}_{2} \cdot \mathrm{H}_{2} \mathrm{O}$ and $\mathrm{FeCl}_{3} \cdot 6 \mathrm{H}_{2} \mathrm{O}$ salts were obtained from Merck, Mumbai, India. Other chemicals and solvents used in the study were of standard quality (AR) grade.

\subsection{Preparation of magnetic starch nanoparticles}

The iron oxide impregnated starch nanoparticles were prepared by following an emulsion crosslinking method. The most common method for production of magnetic nanoparticles involves co-precipitation of ferrous and ferric salts in an alkaline medium with continuous stirring.

$$
2 \mathrm{FeCl}_{3}+\mathrm{FeCl}_{2}+8 \mathrm{NH}_{4} \mathrm{OH} \rightarrow \mathrm{Fe}_{3} \mathrm{O}_{4}+8 \mathrm{NH}_{4} \mathrm{Cl}+4 \mathrm{H}_{2} \mathrm{O} .
$$

In a typical experiment, the 'aqueous phase' was prepared by dissolving a definite amount of starch into $10 \mathrm{~mL}$ of distilled water to form a paste, which was then added to $40 \mathrm{~mL}$ of boiling water to obtain a clear solution. To this solution, $2 \mathrm{~g}$ of iron salts and definite volume of silicon oil were added to form the oil phase. The resulting solutions were mixed with vigorous shaking for $1 \mathrm{~h}$ to form a stable suspension. Now to this suspension $\mathrm{NH}_{4} \mathrm{OH}$ was added and 102.28 $\mathrm{mM}$ epichlorohydrin emulsions are prepared in silicon oil with constant shaking. The crosslinking reaction was allowed to proceed for $5 \mathrm{~h}$ and the nanoparticles so obtained were

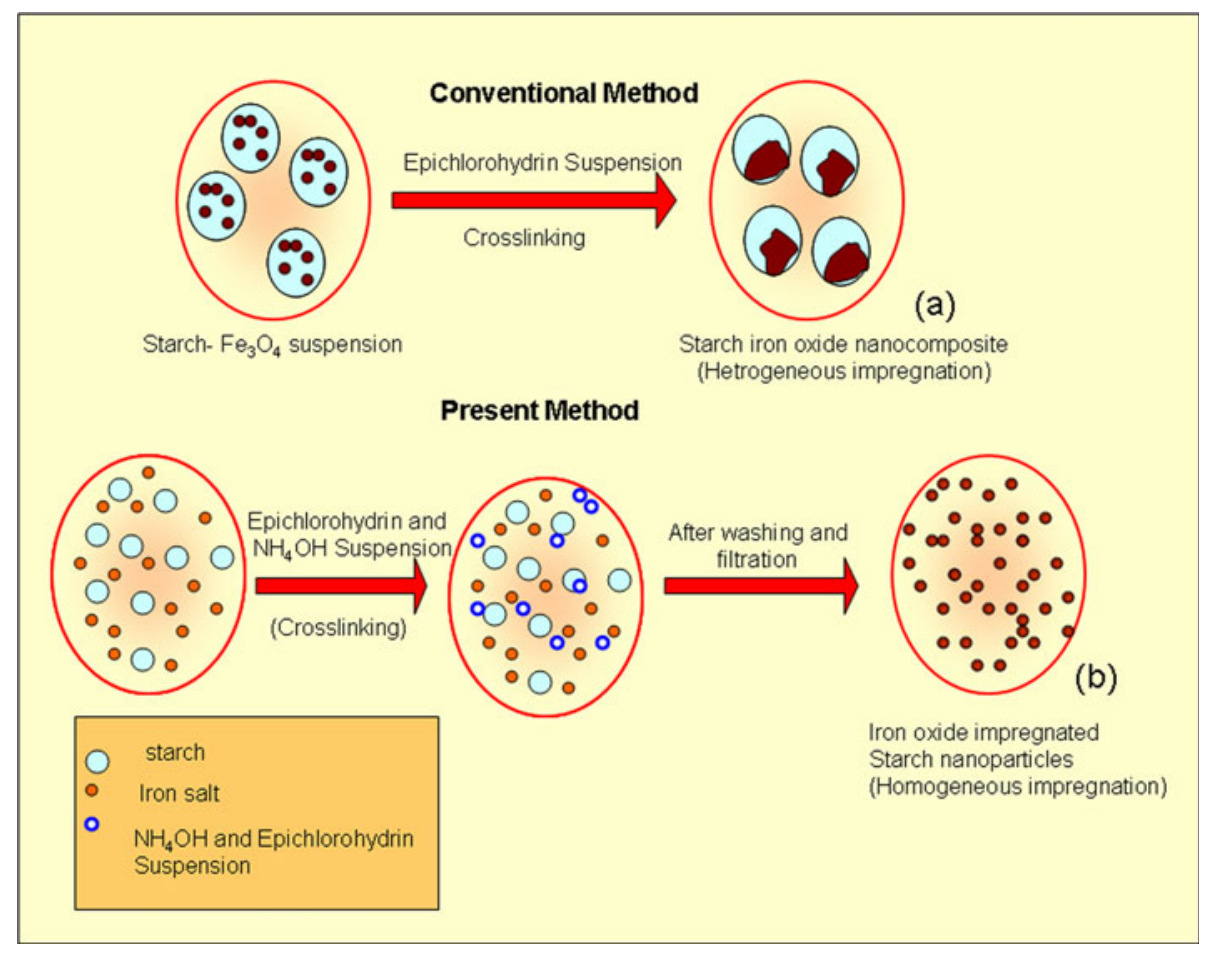

Figure 1. Schematic representation of conventional (a) and present preparation (b) methods of iron oxide impregnated starch nanoparticles. 
successively washed with toluene and acetone. Thoroughly washed nanoparticles were dried and stored in polyethylene bags. It is worth to mention here that in the present study the iron oxide nanoparticles were precipitated inside the starch nanoparticles matrix and, therefore, the method is novel and different from the techniques where a simple blending of nanoparticles is done to achieve the polymer nanocomposite nanoparticles. The superiority of the present method over the conventional ones may be schematically depicted as shown in figure 1.

\section{Characterizations}

\subsection{FTIR spectral analysis}

FTIR spectra of crosslinked nanoparticles were recorded on a FTIR spectrophotometer in the range $400-4000 \mathrm{~cm}^{-1}$ (Perkin-Elmer, 1000 Paragon).

\subsection{X-ray diffraction analysis}

The X-ray diffraction studies of nanoparticles were carried out on a Philips PW1820 powder diffractometer. The diffraction data were collected from 10 to $60,2 \theta$ values with a step size of 0.02 and counting time of $2 \mathrm{~s} \mathrm{step}^{-1}$. The average crystallite size of iron oxide particles was estimated using Scherrer's formula.

\subsection{Transmission electron microscopy analysis}

TEM of the prepared starch particles were recorded to determine internal particle distribution and structural morphology of nanoparticles. Transmission electron microscopy (TEM) was performed by using a Morgagni-268-D transmission electron microscope with an acceleration voltage of $80 \mathrm{kV}$. TEM measurements were done by dispersing a drop of the sample suspension on Formvar-coated $\mathrm{C}$ grids.

\subsection{Vibrating sample magnetometer analysis}

A vibrating-sample magnetometer (VSM) was used to study the magnetic properties of iron oxide impregnated starch nanoparticles at room temperature. The coercivity $\left(H_{\mathrm{c}}\right)$, the remnant magnetizations $\left(M_{\mathrm{r}}\right)$ and the saturation magnetizations $\left(M_{\mathrm{s}}\right)$ of prepared iron oxide nanoparticles were performed by using VSM. The magnetic parameters $\left(H_{\mathrm{c}}, M_{\mathrm{r}}\right.$ and $M_{\mathrm{s}}$ ) of samples were determined from the hysteresis loops produced by VSM.

\subsection{Surface potential (zeta potential) measurements}

The zeta potential of nanoparticles is commonly used to characterize the surface charge property of nanoparticles (Couvreur et al 2002), which is influenced by the composition of the particles and the medium in which they are dispersed. The zeta potential was used to determine whether a charge active material is encapsulated within the centre of the nanocapsule or adsorbed onto the surface. In order to understand the nature of magnetic nanoparticles, zeta potential studies were performed with a digital potentiometer (Model No. 118, El product, Mumbai, India).

\subsection{Water sorption capacity}

The swelling behaviour of polymeric nanoparticles depends on the nature of the solvent and polymer. The swelling properties of nanoparticles affect their usability as a biomaterial in medicine pharmacy and veterinary practices.

Swelling of native starch and iron oxide containing starch nanoparticles was studied by a conventional gravimetric procedure. In a typical experiment, $0 \cdot 1 \mathrm{~g}$ of the nanoparticles were allowed to swell in a definite volume $(10 \mathrm{~mL})$ of phosphate buffer saline ( $\mathrm{pH} 7.4)$ taken in a preweighed sintered glass crucible (pore size, 5-10 $\mu \mathrm{m}$ ) and weighed after a definite period by removing excess phosphate buffer saline by vacuum filtration. The swelling of nanoparticles was monitored continuously up to $15 \mathrm{~min}$ after which no weight gain of swollen nanoparticles was recorded which clearly indicated the arrival of equilibrium swelling conditions. The amount of water imbibed by the nanoparticles was calculated by the following equation

$$
\text { Swelling ratio }=\frac{W_{\mathrm{t}}}{W_{\mathrm{o}}},
$$

where $W_{\mathrm{o}}$ and $W_{\mathrm{t}}$ are the weight of dry and swollen nanoparticles at $o$ and time $t$, respectively.

\subsection{Swelling studies in physiological fluids}

In order to study the swelling of nanoparticles in simulated biological media, the following aqueous fluids $(100 \mathrm{~mL})$ were prepared: saline water $(0.9 \mathrm{~g} \mathrm{NaCl})$, synthetic urine (0.8 g NaCl, $0.10 \mathrm{~g} \mathrm{MgSO}_{4}, 2.0 \mathrm{~g}$ urea, $0.6 \mathrm{~g} \mathrm{CaCl}_{2}$ ), urea $(5 \mathrm{~g})$ and D-glucose $(5 \mathrm{~g})$.

\subsection{Effect of $\mathrm{pH}$}

The effect of $\mathrm{pH}$ on swelling of the nanoparticles was studied by preparing solution in the studied range of $\mathrm{pH}$ between 1.8 and 8.6 by adding $0.1 \mathrm{M} \mathrm{HCl}$ and $0.1 \mathrm{M} \mathrm{NaOH}$ to bring the desired $\mathrm{pH}$ values. The $\mathrm{pH}$ was determined on a digital pH meter (Systronics, No. 362, Ahmedabad, India).

\subsection{Statistical analysis}

All the experiments were done at least thrice and data and figures have been shown along with respective S.D. and error bars, respectively. 


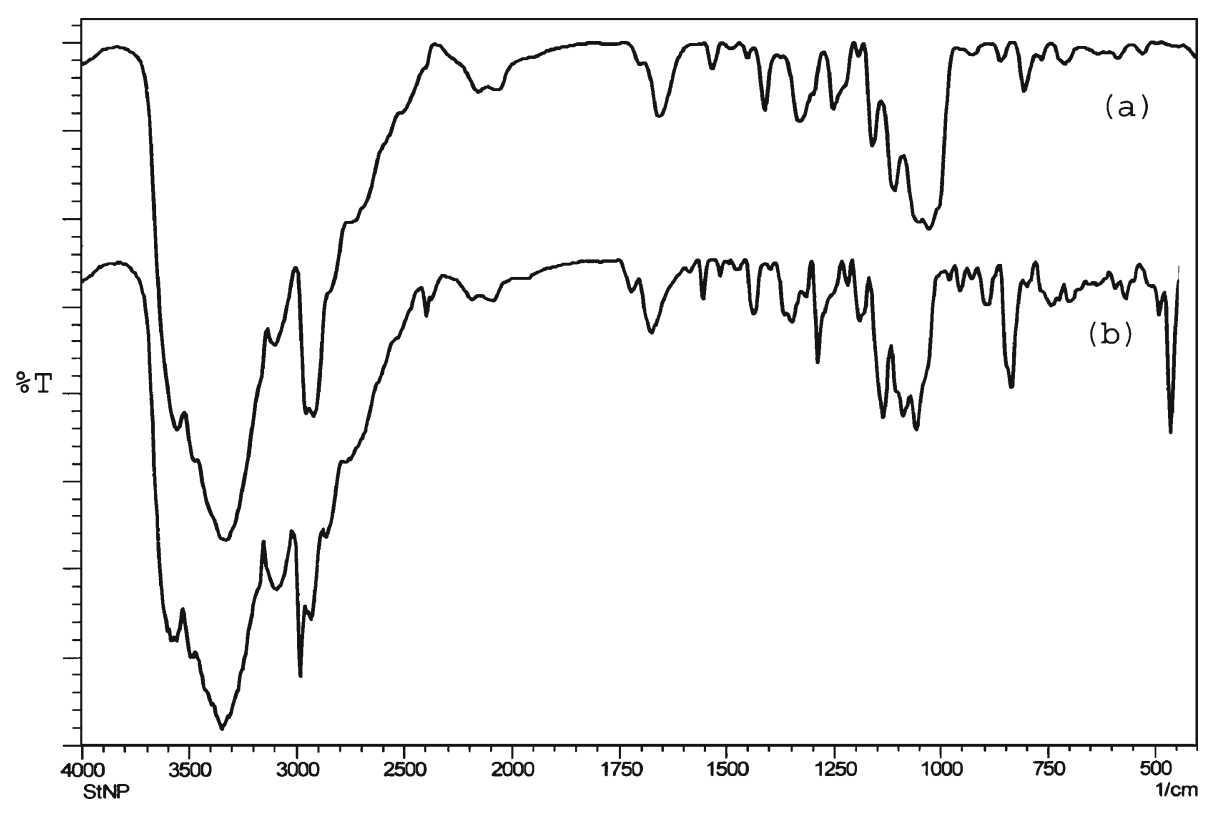

Figure 2. FTIR spectra of (a) starch nanoparticles and (b) iron oxide impregnated starch nanoparticles.

\section{Results and discussion}

\subsection{FTIR spectroscopy}

FTIR spectra of native starch nanoparticles and iron oxide impregnated starch nanoparticles are shown in figure $2 a$ and $b$, respectively which confirm the presence of different characteristic functional groups of the constituents of nanocomposite nanoparticles. In the IR spectra the peaks observed at $3581 \mathrm{~cm}^{-1}$ indicates the presence of starch due to its $\mathrm{O}-\mathrm{H}$ stretching vibration. Additional multiple bands appear at lower frequency in the range $3000-3500 \mathrm{~cm}^{-1}$ at the expense of the starch hydroxyl groups. The spectra also depict $\mathrm{C}-\mathrm{H}$ stretching at $2920 \mathrm{~cm}^{-1}, \mathrm{O}-\mathrm{H}$ bending at $1259 \mathrm{~cm}^{-1}, \mathrm{C}-\mathrm{O}$ stretching at $1026 \mathrm{~cm}^{-1}$ and a prominent C-O-C stretching of the glycosides bonds at $1105 \mathrm{~cm}^{-1}$, typical for starch. The spectra (b) also show the presence of iron oxides as evident from the peaks observed at $526-418 \mathrm{~cm}^{-1}$. Similar types of results have also been reported elsewhere (Shrivastava and Bajpai 2005).

\subsection{Powder X-ray diffraction (XRD) analysis}

The crystalline nature of iron oxide impregnated starch nanoparticles was determined by using XRD analysis, which is an essential tool for the determination of crystallinity of the material. The well defined X-ray diffraction patterns indicate the formation of highly crystalline iron oxide nanoparticles. XRD pattern of the prepared native starch nanoparticles and iron oxide impregnated starch nanoparticles are shown in figures $3 a$ and $b$, respectively. X-ray diffraction patterns of the starch nanoparticles is shown in figure $3 \mathrm{a}$ which indicates the characteristic peak of starch at $20 \cdot 2^{\circ}$, whereas the appearance of two characteristics peaks at $21.4^{\circ}, 37 \cdot 1^{\circ}$, of iron oxide indicates the impregnation of iron oxide on starch nanoparticles, and the mean grain size of the iron oxide impregnated starch nanoparticles has been calculated using DebyeScherrer formula (Bajpai and Bundela 2008) as shown in (2):

$$
d=\frac{k \lambda}{\beta \cos \theta},
$$

where $d$ is the mean grain size, $k$ the shape factor (0.9), $\beta$ the broadening of the diffraction angle and $\lambda$ the diffraction wavelength $(1.54 \AA)$. The estimated average grain size of iron oxide impregnated starch nanoparticles was found to be $6.8 \mathrm{~nm}$.

The amorphous and crystalline nature of the iron oxide impregnated starch nanoparticles were determined by $\%$ crystallinity. The numerical formula to calculate the percent crystallinity of nanoparticles is given in the following equation:

$$
X_{\mathrm{c}}(\%) \frac{A_{\mathrm{c}}}{A_{\mathrm{a}}+A_{\mathrm{c}}} \times 100,
$$

where $A_{\mathrm{c}}$ and $A_{\mathrm{a}}$ are the areas of crystalline and amorphous phases, respectively (Gupta and Bajpai 2010). The crystallinity of the material has been calculated by the formula given in (3). The \% crystallinity of native starch was found to be 43.8 while for iron oxide impregnated starch nanoparticles the $\%$ crystallinity was about 67.5 . These results are quite obvious as being an amorphous polymer the starch shows lesser crystalline nature than the material containing iron oxide in combination with starch.

\subsection{TEM analysis}

TEM provides a powerful technique to determine size and morphology of the nanoparticles. The size of particles 
usually refers to the total diameter of the particles and is a key property to decide the suitability of the carrier to in vivo applications. Since the smallest diameter of capillaries in the body is $4000 \mathrm{~nm}$, larger particles will be mainly captured and withheld in the lungs. Particles with larger sizes and /or aggregation of smaller particles thus may be trapped, causing emboli within the capillaries of the lungs (Kreuter 1983). Depending on their magnetic energy, most nanoparticles have a tendency to aggregate, thus reducing their surface charge. This may lead to precipitation which could be dangerous if these particles are injected intravenously.
Therefore, it is important to know the size of the nanoparticles to be proposed for biomedical application. Most intravenously applied nanoparticles are recognized as "foreign" in the body system and are eliminated immediately through macrophages of the mononuclear phagocytosis system. Particles smaller than $4000 \mathrm{~nm}$ are taken up through cells of the reticulo-endothelial system, mainly in the liver and spleen (Muller et al 1997). While it is more likely that small particles up to $100 \mathrm{~nm}$ will be phagocytosed through liver cells, there is a tendency for particles larger than $200 \mathrm{~nm}$ to be filtered by the venous sinuses of the spleen. If particles

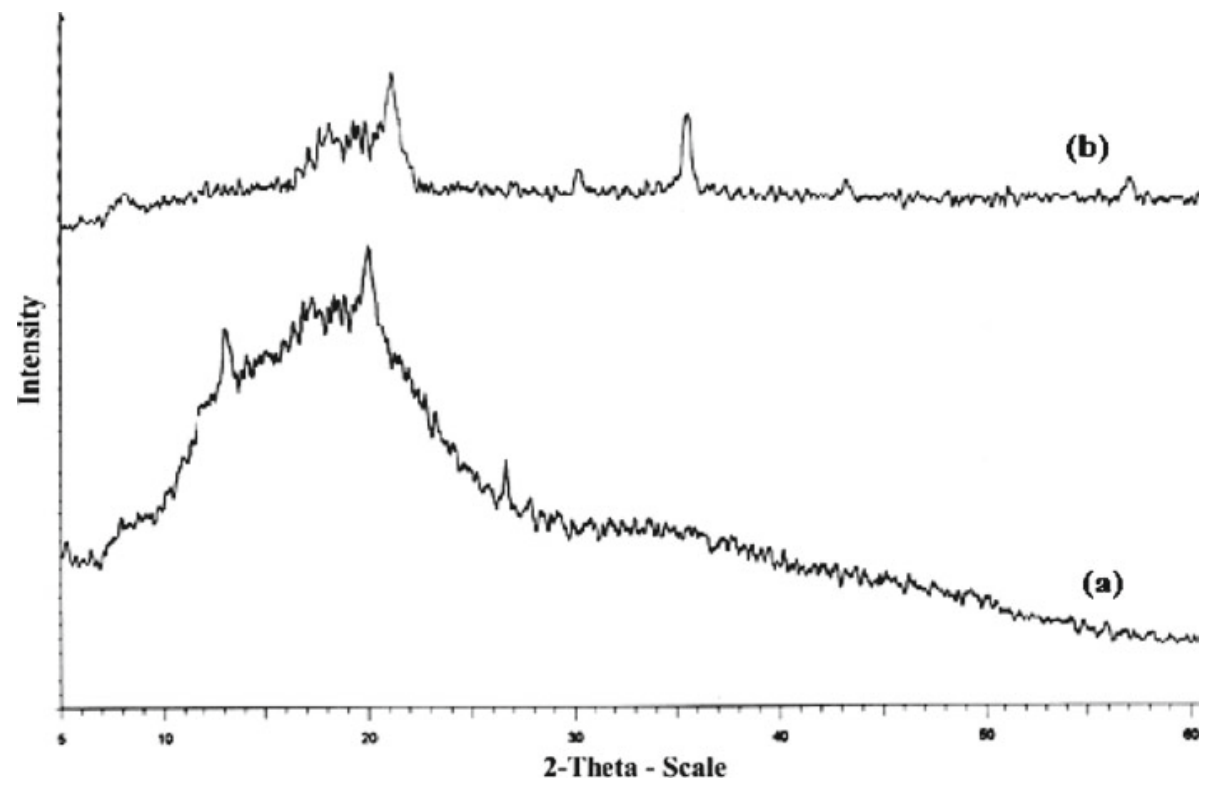

Figure 3. (a) X-ray diffraction pattern of starch nanoparticles and (b) iron oxide impregnated starch nanoparticles.

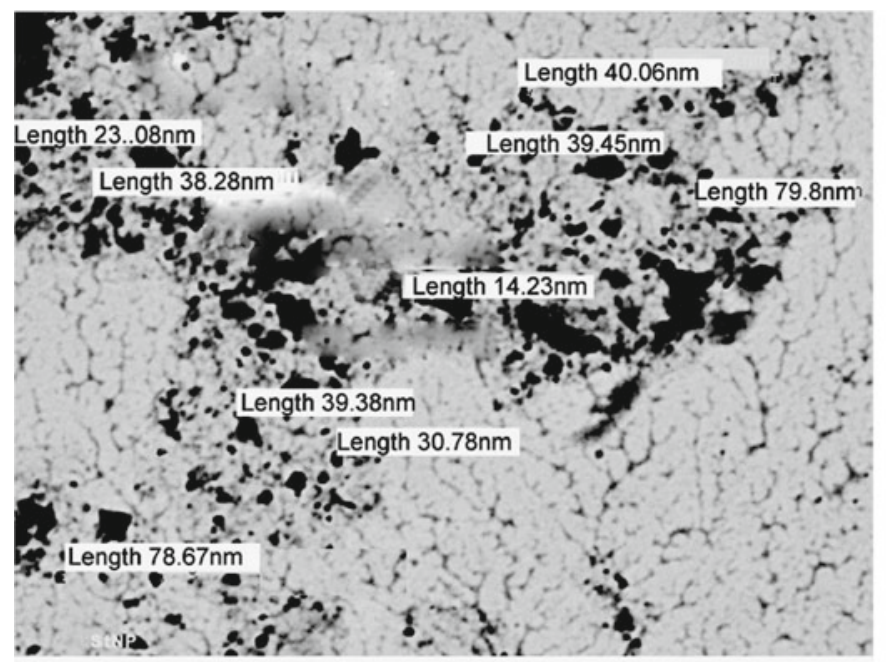

(a)

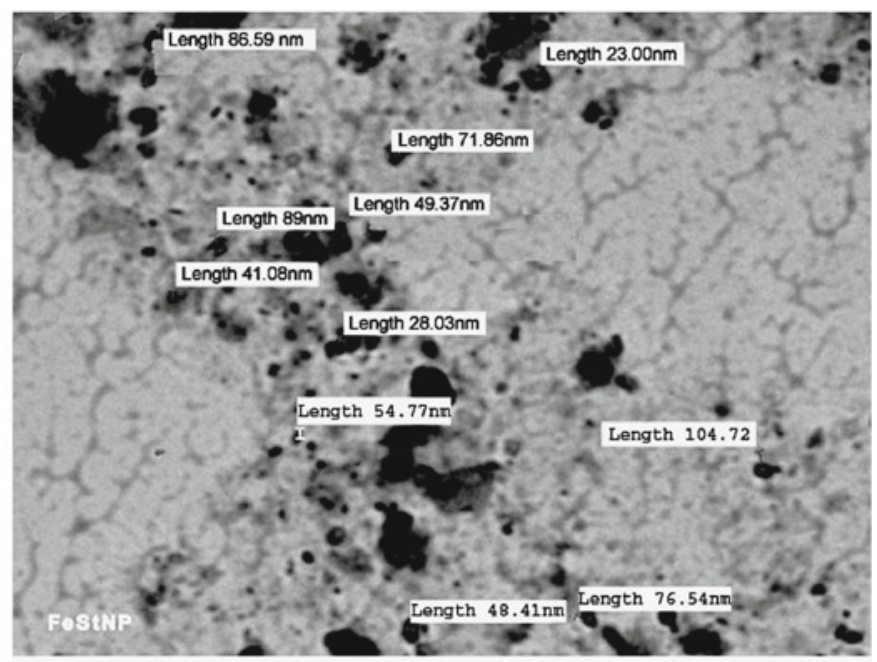

(b)

Figure 4. TEM images of (a) iron oxide impregnated starch nanoparticles and (b) crossliked starch nanoparticles. 
between 30 and $100 \mathrm{~nm}$ are intravenously applied, the liver eliminates the larger particles faster from the bloodstream compared to the smaller sizes. Thus, the larger the particles, the shorter is their plasma half life period. Thus the larger particles will be removed by cells capable of phagocytosis; whereas smaller particles can be removed by all types of cells through pinocytosis (all cells are capable of pinocytosis).

In order to investigate size and morphology of prepared starch nanoparticles and iron oxide impregnated starch nanoparticles, transmission electron micrograph (TEM) images were recorded as shown in figures $4(\mathrm{a}, \mathrm{b})$, respectively which represent the image of nanoparticles clusters and single nanoparticles. It is clear from the images $(a, b)$ the size of the aggregated nanoparticles was in the range of 20-80 nm and while those of single and non-aggregated nanoparticles in the range of 7-9 nm. The image (a) which represents morphology of the iron oxide impregnated starch nanoparticles, however, indicates for aggregation of nanoparticles within the starch matrix. The reason for the observed aggregation may be attributed to the fact that starch is a hydrophilic biopolymer and may show a tendency to get adsorbed onto the surfaces of the iron oxide nanoparticles. Thus starch nanoparticles may interact with various iron oxide nanoparticles and may result in agglomeration of iron oxide nanoparticles.

\subsection{Vibrating sample magnetometry (VSM) analysis}

The magnetic properties of magnetic polymeric nanoparticles were studied using a vibrating sample magnetometer. Figure 5 shows a typical magnetization $(M)$ vs the applied magnetic field $(H)$ curve. The saturation magnetization of synthetic magnetic particles was found to be $58 \mathrm{emu} / \mathrm{g}$ at $300 \mathrm{~K}$. The value obtained was lower than the reported values of 90-100 emu/g for magnetite $\left(\mathrm{Fe}_{3} \mathrm{O}_{4}\right)$ nanoparticles (Morales et al 2005) and may be attributed to the fact that

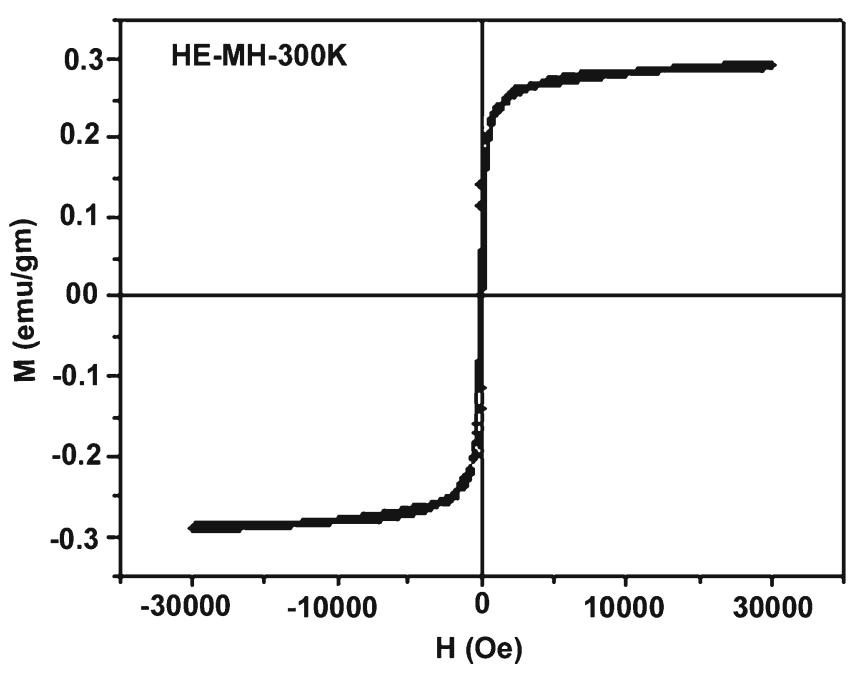

Figure 5. Hysteresis curve of iron oxide impregnated starch nanoparticles obtained by VSM measurements.
Table 1. Zeta potential of iron oxide impregnated starch nanoparticles at various $\mathrm{pH}$.

\begin{tabular}{cccc}
\hline EMF (mV) & $\begin{array}{c}\text { EMF (mV) } \\
\text { pH (buffer solution) }\end{array}$ & $\begin{array}{c}\text { EMF (mV) (iron oxide } \\
\text { impregnated starch } \\
\text { nanoparticles) }\end{array}$ \\
\hline 1.8 & 293.9 & 299.3 & 305 \\
7.4 & -30.3 & 11.6 & 10 \\
8.6 & -87.6 & -80 & -55 \\
\hline
\end{tabular}

below a critical size, nanocrystalline magnetic particles may be single domain and show the unique phenomenon of superparamagnetism (Chia et al 2006). The magnetization curve also reveals that the value of $M_{\mathrm{s}}$ increases with decrease in temperature and superparamagnetism behaviour of magnetic particles was maintained.

\subsection{Surface potential measurements}

The surface charge possessed by organic-inorganic hybrid nanoparticles is related to the electrical potential of the shear plane of the double layer which is measured through the electrophoretic mobility (MaaBen et al 1993). The magnitude and type of the zeta potential of nanoparticles is an important property in deciding stability of the suspension and adsorption properties of biomolecules in vivo. If the zeta potential is lower than a given critical value of the particulate system, aggregation and precipitation of the particles occurs. The surface charge also plays an important role during endocytosis. There should be a slower uptake for negatively charged particles due to the negative rejection effect of the negatively charged cell membranes. However, the endocytosis index in vitro is minimal with a zeta potential close to zero (Kissel and Roser 1991). In contrast, phagocytosis is increased with a higher surface charge independent of whether the charge is negative or positive. The higher the surface charge, the shorter is the residence time of nanoparticles in the circulatory system (Chouly et al 1996).

In the present study, the zeta potential measurements are summarized in table 1 which clearly indicates that upon loading of iron oxide onto starch nanoparticles surface a net increase in positive potential was noticed. The zeta potential value at $\mathrm{pH} 1.8$ is $305 \mathrm{mV}$; at $\mathrm{pH} 7.4$ is 10 and at $\mathrm{pH}$ 8.6 is $-55 \mathrm{mV}$. The decrease in potential with an increasing $\mathrm{pH}$ indicates that at lower $\mathrm{pH}$ the iron oxides were in $\left(\mathrm{FeOH}_{2}^{+}\right)$form and showed a positive potential (DouziechEyrolles et al 2007), while negative potential values were observed at higher range of $\mathrm{pH}$ which is due to the anionic form $\left(\mathrm{FeO}^{-}\right)$of iron oxide. Similar type of results has been reported elsewhere (Guo et al 2009).

\subsection{Effect of chemical compositions}

The influence of the chemical composition of the iron oxide impregnated starch nanoparticles on their swelling ratio was 


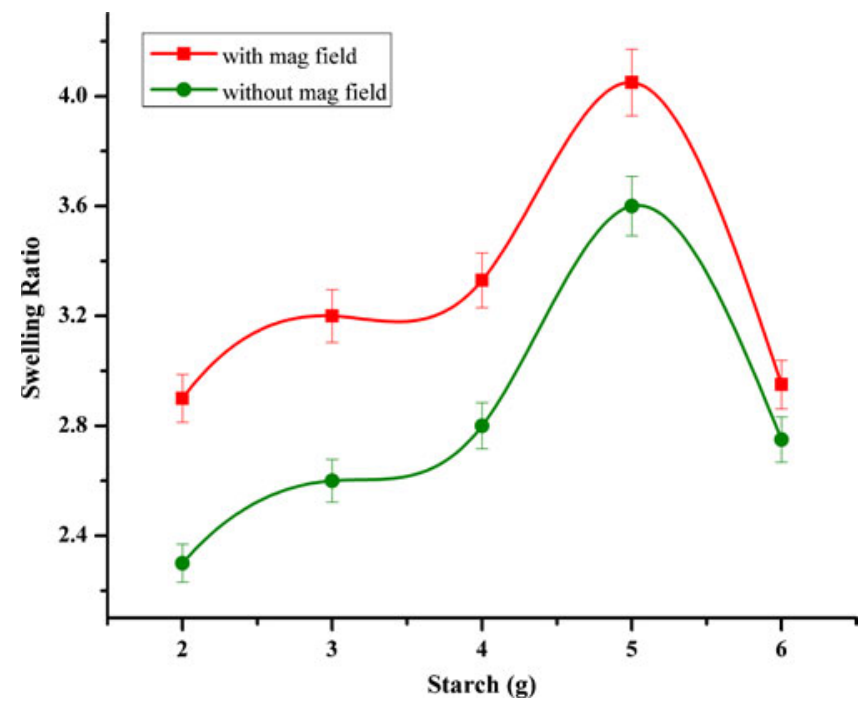

Figure 6. Effect of varying amounts of starch on swelling ratio of nanoparticles of definite composition (epichlorohydrin $=$ 102. $28 \mathrm{mM}$, ferrous and ferric salt $=2 \mathrm{~g}$ ).

investigated by varying the amounts of starch and crosslinker (epichlorohydrin) in the feed mixture as discussed below.

\subsection{Effect of amount of starch}

The effect of increasing starch content on the swelling characteristics of the iron oxide impregnated starch nanoparticles has been investigated by varying the amount in the range $2 \cdot 0-6.0 \mathrm{~g}(1: 5 \cdot 5-2: 5 \cdot 2 \mathrm{wt}$ ratio $\mathrm{g} / \mathrm{g}$ of starch to epichlorohydrin) in the feed mixture in the presence and absence of magnetic field. The results are shown in figure 6, which clearly indicate that the swelling of starch initially increases up to $5.0 \mathrm{~g}$ (1:2.9 wt ratio $\mathrm{g} / \mathrm{g}$ of starch to epichlorohydrin) of starch content and thereafter decreases with further increase in the amount of starch content. The results may be attributed to the fact that starch is a hydrophilic biopolymer and its increasing amount in the particles will obviously enhance the hydrophilicity of the nanoparticles and, thus, an increase in the swelling ratio is expected. However, beyond 5.0 $\mathrm{g}$ (1:2.9 wt ratio $\mathrm{g} / \mathrm{g}$ of starch to epichlorohydrin) of starch content, the observed decrease in swelling ratio may be due to the enhanced compactness of the particles and greater interaction between macromolecular chains of starch nanoparticles. The results also indicate that swelling of nanoparticles in magnetic field is higher than that in the absence of magnetic field. The results can be explained by the fact that due to the applied magnetic field, the magnetic moments of impregnated iron oxide nanoparticles tend to get aligned with the external magnetic field and while doing so, they produce a motion of macromolecular chain in the starch matrix. Due to the mobility of iron oxide nanoparticles the macromolecule chains of starch get relaxed and facilitate inclusion of water molecules into the biopolymer matrix. This clearly results in an enhanced swelling.
It is important to mention here that the influence of magnetic field on the swelling of native starch nanoparticles was also investigated and it was found that no increase in swelling was shown in the presence of magnetic field. The observed results are quite clear as starch is diamagnetic in nature. The applied magnetic field will not have any effect on the swelling capacity of the nanoparticles.

\subsection{Effect of crosslinker (epichlorohydrin)}

A large number of crosslinker are known for crosslinking of starch among which phosphoryl chloride, epichlorohydrin and tri-sodium tri-metaphosphate are the most common ones (Bajpai and Bhanu 2007). In the present study, however, epichlorohydrin was used to crosslink starch (8:23-5.9:8.4 wt ratio $\mathrm{g} / \mathrm{g}$ of epichlorohydrin to starch) and employed in the concentration range $24.4-127.85 \mathrm{mM}$ in the feed mixture. The results reveal that up to $102.28 \mathrm{mM}(1.7: 3.2 \mathrm{wt}$ ratio $\mathrm{g} / \mathrm{g}$ epichlorohydrin to starch) of added crosslinker, the water sorption of nanoparticles constantly increases while beyond this concentration a drop in swelling capacity of nanoparticles is noticed. The observed increase could be attributed to the fact that epichlorohydrin is a low molecular weight crosslinking agent of starch, and it crosslinks by reacting with the hydroxyl groups of starch at its two terminals. Thus, a crosslinked starch network could be imagined as an ultrahigh molecular weight starch molecule that contains wide pore size in its structure and, therefore, possesses an abnormal capacity of accommodating water molecules into the network. Thus, capacity to imbibe increasing number of water molecules results in an increased swelling which is clearly shown in figure 7 .

The decrease observed beyond 102.28 mM (1.7:3.2 wt ratio $\mathrm{g} / \mathrm{g}$ epichlorohydrin to starch) of epichlorohydrin may

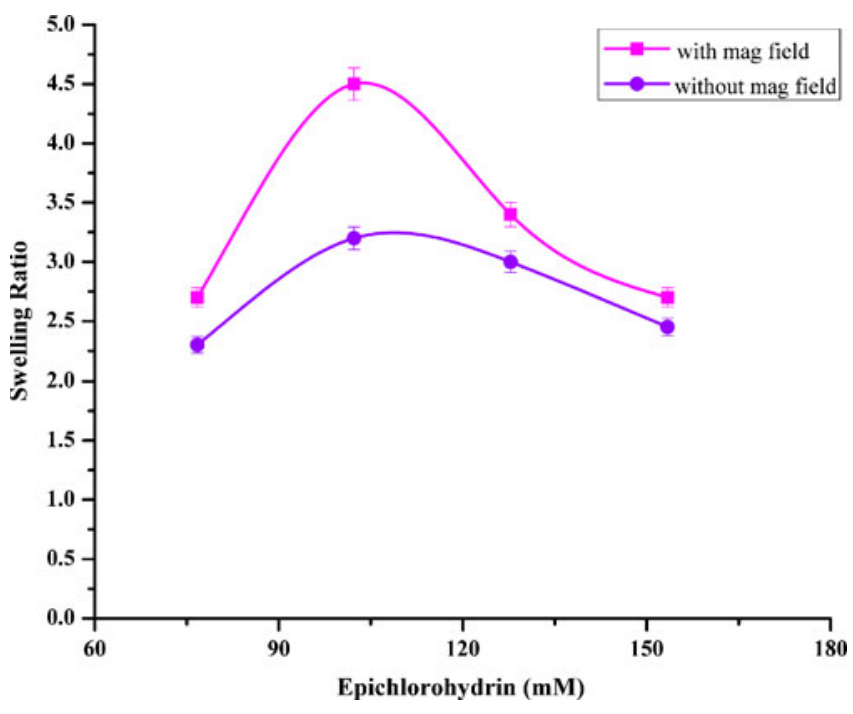

Figure 7. Effect of varying amounts of epichlorohydrin on swelling ratio of nanoparticles of definite composition ( $\operatorname{starch}=5 \mathrm{~g}$, ferrous and ferric salt $=2 \mathrm{~g}$ ). 
be explained by the reason that much higher crosslinker content in the nanoparticles matrix reduces the free volumes of nanoparticles network accessible to the penetrant water molecules and consequently results in a fall in the swelling capacity. Similar type of results has been reported by other workers also (Chairam and Somsook 2008). The results also indicate that magnetic field also enhances the swelling of nanoparticles which has been explained earlier.

\subsection{Effect of simulated biological fluids}

The effect of the nature of biological fluids on the swelling of crosslinked starch nanoparticles has been investigated by performing swelling experiments in various simulated physiological fluids. The results are depicted in figure 8, which show an increase or decrease in swelling behaviour of the iron oxide impregnated starch nanoparticles. The effect

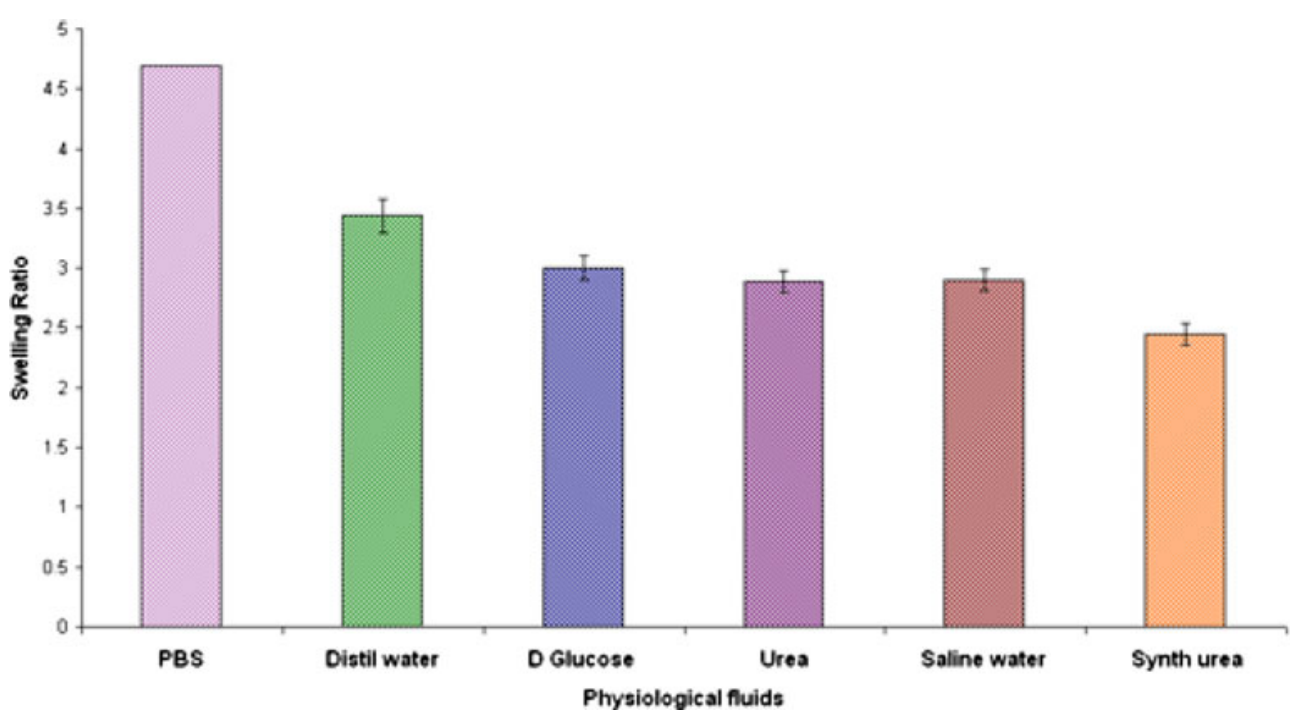

Figure 8. Effect of physiological fluids on iron oxide impregnated starch nanoparticles of definite amount of starch $=5 \mathrm{~g}$, epichlorohydrin $=102 \cdot 28 \mathrm{mM}$, ferrous and ferric salt $=2 \mathrm{~g}$.

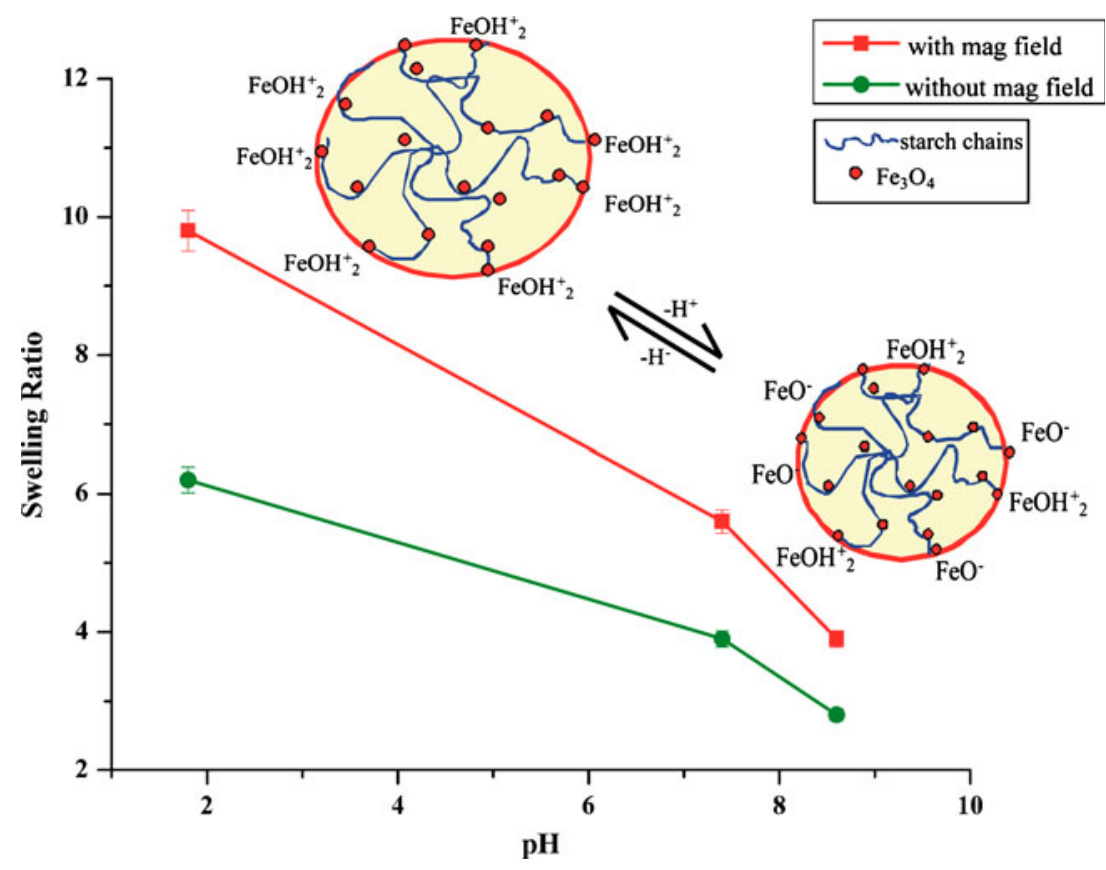

Figure 9. Effect of $\mathrm{pH}$ on iron oxide impregnated starch nanoparticles of definite amount of starch $=5 \mathrm{~g}$, epichlorohydrin $=102.28 \mathrm{mM}$, ferrous and ferric salt $=2 \mathrm{~g}$. 
of biological fluids was examined by performing swelling experiments in the presence of urea, D-glucose and potassium iodide, saline water and artificial urine. The results are summarized in figure 8 which clearly shows that the presence of solute suppresses the swelling ratio of these nanoparticles. The possible reason for the observed lower swelling of particles in these fluids may be the presence of salt ions in the release medium which lowers the osmotic pressure of the swelling system thus resulting in a lower swelling of impregnated starch nanoparticles.

\subsection{Effect of $p H$}

$\mathrm{pH}$ responsive macromolecular devices have been most frequently used to design controlled release formulations for oral administration which remains the most clinically acceptable way of drug delivery (Lüebbe et al 2001). In the present investigation, the influence of $\mathrm{pH}$ on the swelling ratio of starch nanoparticles has been studied by adjusting the $\mathrm{pH}$ of swelling medium at 1.8, 7.4 and 8.6. The results are shown in figure 9 , which clearly reveal that the swelling ratio of particles constantly decreases in the whole studied range. The decrease observed in the swelling ratio of the particles with increasing $\mathrm{pH}$ may be explained as below.

Since starch is non-ionic in nature, the change in $\mathrm{pH}$ of the swelling medium does affect the charge profiles of the iron oxide nanoparticles in an aqueous medium which eventually influences the water sorption capacity of the nanocomposite. What actually happens is that at low $\mathrm{pH}$ of $1 \cdot 8, \mathrm{FeOH}$ groups of the iron oxide nanoparticles present on the surfaces and bulk of starch nanoparticles matrix get protonated thus producing $\mathrm{FeOH}_{2}^{+}$ions which is due to mutual repulsion tending to relax the starch chains and result in higher swelling ratio. As the $\mathrm{pH}$ of the medium increases, the existing repulsion forces goes on decreasing which consequently produces a decreasing swelling of the nanoparticles. This fall in water sorption capacity continues till the zero-point charge of iron oxide which is reported to be in the range 7-8. At the point of zero charge an equal number of $\mathrm{FeOH}_{2}^{+}$ and $\mathrm{FeOH}^{-}$ionic groups exist which causes a minimum repulsion between the macromolecular chains of starch and result in a minimum swelling. A slight increase is, however, observed upon further increasing $\mathrm{pH}$ of the medium which may be attributed to the reason that due to the repulsion between the anionic groups $\mathrm{FeOH}^{-}$swelling ratio slightly increases. The explanation of $\mathrm{pH}$ effect on swelling behaviour has been presented by a hypothetical model in figure 9 . Also note that greater swelling is shown by nanocomposite particles in the presence of applied magnetic field.

\subsection{Effect of temperature}

In the present study, the temperature of release medium was varied in the range $10-45^{\circ} \mathrm{C}$ and its effect on swelling ratio was investigated. The results are displayed in figure 10 which indicates that with increasing temperature, the swelling of

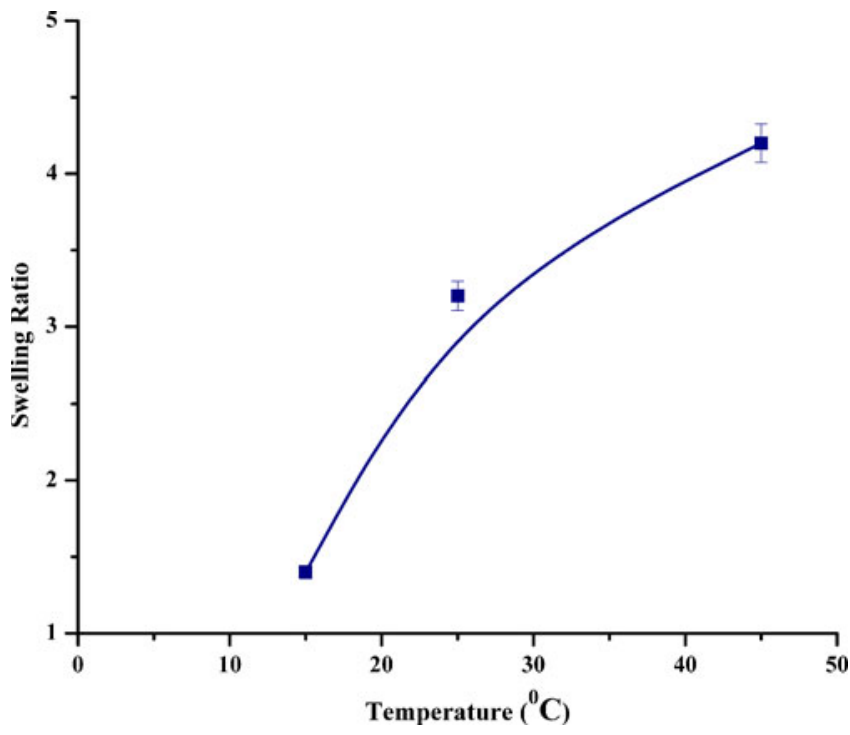

Figure 10. Effect of temperature on iron oxide impregnated starch nanoparticles of definite amount of starch $=5 \mathrm{~g}$, epichlorohydrin $=102 \cdot 28 \mathrm{mM}$, ferrous and ferric salt $=2 \mathrm{~g}$.

nanoparticles increases in the whole studied range. The observed increase in the swelling of iron oxide impregnated starch nanoparticles can be explained by the fact that a rise in temperature enhances rate of water diffusion and segmental mobility of macromolecular chains which combined results in a greater degree of swelling.

\section{Conclusions}

In the present study, we have demonstrated a novel method for synthesizing homogeneously dispersed iron oxide impregnated superparamagnetic starch nanoparticles. The magnetite nanoparticles were synthesized by using the in situ precipitation of magnetic iron oxide nanoparticles by a wet chemical method. The nanoparticles were characterized by various analytical techniques, such as FTIR spectroscopy, TEM and XRD analyses which confirm the in situ impregnation of nanosized iron oxide within the matrix of starch nanoparticles. The polymeric nanoparticles clearly show the presence of characteristic groups of starch and iron oxide as confirmed by their FTIR spectra. XRD and TEM of the nanoparticles provide information about their semicrystalline nature and nanosize of the particles, respectively.

The swelling behaviour of nanocomposite nanoparticles is enhanced when the starch content increases from 2.0 $5.0 \mathrm{~g}$ while at $6.0 \mathrm{~g}$ of starch content the degree of swelling decreases. Likewise, when the concentration of crosslinker increases from $24.4 \mathrm{mM}$ to $102.28 \mathrm{mM}$, the quantity of water imbibed by the nanoparticles increases while beyond $102.28 \mathrm{mM}$ of crosslinker concentration the extent of swelling decreases. The nanoparticles also show a lower swelling in simulated biological fluids. It is also found that in acidic $\mathrm{pH}$ the nanoparticles show an enhanced 
swelling while with increase in $\mathrm{pH}$ of the swelling bath, the water sorption decreases. In the case of rising temperature the swelling ratio constantly increases. The prepared nanoparticles exhibit superparamagnetism which is a significant property for a variety of biomedical applications. It is also observed that the swelling of nanoparticles is significantly enhanced by the application of external magnetic field. Thus the present swelling system may be helpful in designing targeted drug delivery carriers.

\section{References}

Arruebo M, Fernandez-Pacheco R and Ibarra M R 2007 Nanotoday 222

Bajpai A K and Bhanu S 2007 J. Mater. Sci. Mater. Med. 181613

Bajpai A K and Bundela H 2008 eXpress Polym. Lett. 2201

Balaban R S, Koretsky A P and Dunbar C E 2003 Blood 102867

Chairam S and Somsook E 2008 J. Magn. Magn. Mater. 320 2039

Chia C H, Zakaria S, Ahmad S, Abdullah M H and Jani S M 2006 Am. J. Appl. Sci. 31750

Chouly C et al 1996 J. Microencapsulation 13245

Conte L L, Nitin N and Bao G 2005 Nanotoday 832

Corre D, Bras J and Dufrense A 2010 Starch Nanoparticles 111139

Couvreur P, Barratt G, Fattal E, Legrand P and Vauthier C 2002 Crit. Rev. Ther. Drug Carrier Syst. 1999

Douziech-Eyrolles L, Marchais H, Hervé K, Munnier E, Linassier C, Dubois P and Chourpa I 2007 Int. J. Nanomed. 2541

Fang J and Fowler P 2002 Food Agric. Environ. 182

Gupta R and Bajpai A K 2010 J. Biomater. Sci. Polym. Ed. 1

Gomez-Lopera S, Plaza, R C and Delgado A V J 2001 Coll. Interf. Sci. 24040
Guo S J, Li D, Zhang L and Wang E K 2009 Biomaterials 30 1881

Honda H, Kawabe A, Shinkai M and Kobayashi T 1998 J. Ferment Bioeng. 86191

Kim K D et al 2003 Chem. Mater. 543

Kissel T and Roser M 1991 Proceeding of the international symposium on controlled release of bioactive materials, San Diego 18 275

Kreuter J 1983 Pharm. Acta Helv. 58196

Kumar C S S R 2005 Biofunctionalization of nanomaterials (Weinheim: Wiley-VCH) 1 p. 1468

Kusaka M, Takegami K, Sudo A, Yamazaki T, Kawamura J and Uchid A 2002 J. Orthop. Sci. 7354

Lehr C M, Schaefer U F, Wenz G, González D B, Ortega-Vinuesa J L, Loretz B, Stauner T and Santander-Ortega M J 2010 J. Control. Rel. 14185

Liu X, Yueping G, Zhiya M and Liu H 2004 Langmuir 2010278

Lüebbe A S, Alexiou C and Bergemann C 2001 J. Surg. Res. 95 200

MaaBen S, Fattal E and Muller R H 1993 STP Pharm. Sci. 311

Morales M A, Jain T K and Labhasetwar V 2005 J. Appl. Phys. 971

Mohanraj V J and Chen Y 2006 J. Pharm. Res. 5561

Muller R H et al 1997 Scientific and clinical applications of magnetic carriers (eds) U Hafeli et al (New York: Plenum Press)

Nidhin M, Indumathy R, Sreeram K J and Balachandran U N 2008 Bull. Mater. Sci. 31936

Parikh R H, Parikh J R, Dubey R R, Soni H N and Kapadia K A 2003 J. Pharm. Sci. 131

Roullin V G, Deverre J R, Lemaire L, Hindre F, Venier-Julienne M C and Vienet R 2002 Eur. J. Pharm. Biopharm. 59293

Saboktakin M R, Maharramov A and Ramazanov M A 2009 Carbohyd. Polym. 78292

Shrivastava J and Bajpai A K 2005 Polym. Int. 541524 